\title{
Single low-dose exposure to cow's milk at diagnosis accelerates cow's milk allergic infants' progress on a milk ladder programme
}

\author{
Yvonne d'Art $\mathrm{M}^{1}$, Lisa Forristal ${ }^{2}$, Aideen Byrne ${ }^{3}$, John Fitzsimons ${ }^{3}$, Ronald Van Ree ${ }^{4}$, \\ Audrey DunnGalvin ${ }^{2}$, and Jonathan O'B Hourihane ${ }^{1}$ \\ ${ }^{1}$ University College Cork School of Medicine \\ ${ }^{2}$ University College Cork School of Applied Psychology \\ ${ }^{3}$ Childrens Health Ireland Dublin Ireland \\ ${ }^{4}$ Amsterdam UMC Locatie AMC
}

November 5, 2021

\begin{abstract}
Background Cow's milk protein allergy (CMPA) is one of the most common food allergies in infancy. Most infants with CMPA tolerate baked milk from diagnosis and gradually acquire increased tolerance. Nevertheless, parents often display significant anxiety about this condition and a corresponding reluctance to progress with home introduction of dairy due to concerns about possible allergic reactions. Objective: To evaluate the impact on gradual home introduction of foods containing cows milk after a supervised, single low dose exposure to whole milk at time of diagnosis. Methods Infants less than 12 months old, referred with suspected IgE-mediated cow's milk allergy were recruited to an open-label randomised, controlled trial of intervention - a single dose of fresh cow's milk, using the validated dose of milk that would elicit reactions in $5 \%$ of CMPA subjects - the ED 05 - vs routine care. Both groups implemented graded exposure to CM (using the 12 step MAP Milk Tolerance Induction Ladder), at Home. Parents completed food allergy quality of life and State and Trait Anxiety Inventories (STAI). Main outcome measures were milk ladder position at 6 months and 12 months post randomisation. Results: Sixty patients were recruited, 57 (95\%) were followed to 6 months. By 6 months $27 / 37$ (73\%) intervention subjects had reached step 6 or above on the milk ladder compared to $10 / 20(50 \%)$ control subjects $(\mathrm{p}=0.048)$. By 6 months $11 / 37(30 \%)$ intervention subjects had reached step 12 (ie drinking unheated cow's milk) compared to $2 / 20(10 \%)$ of the controls $(\mathrm{p}=0.049)$. Twelve months post randomisation $31 / 36(86 \%)$ of the intervention group and 15/19(79\%) of the control group were on step 6 or above. However, $24 / 37(65 \%)$ of the intervention group were at step 12 compared to 7/20 (35\%) of the control group ( $\mathrm{p}=0.03)$. Maternal STAIs were significantly associated with their infants' progress on the milk ladder and with changes in skin prick test and spIgE levels at 6 and 12 months. Conclusion This study demonstrates the safety and effectiveness of introduction of baked milk implemented immediately after diagnosis of cows milk allergy in a very young cohort. A supervised single dose of milk at the ED 05 significantly accelerates this further, probably by giving parents the confidence to proceed. Maternal anxiety generally reflects infants' progress towards completion of the milk ladder, but pre-existing high levels of maternal anxiety are associated with poorer progress.
\end{abstract}

\section{Hosted file}

d\&apos;art 2021.11.01.docx available at https://authorea.com/users/444686/articles/544377single-low-dose-exposure-to-cow-s-milk-at-diagnosis-accelerates-cow-s-milk-allergicinfants-progress-on-a-milk-ladder-programme 